\title{
JMcmprsk: An R Package for Joint Modelling of Longitudinal and Survival Data with Competing Risks
}

\author{
by Hong Wang, Ning Li, Shanpeng Li, and Gang Li
}

\begin{abstract}
In this paper, we describe an R package named JMcmprsk, for joint modelling of longitudinal and survival data with competing risks. The package in its current version implements two joint models of longitudinal and survival data proposed to handle competing risks survival data together with continuous and ordinal longitudinal outcomes respectively (Elashoff et al., 2008; Li et al., 2010). The corresponding $\mathrm{R}$ implementations are further illustrated with real examples. The package also provides simulation functions to simulate datasets for joint modelling with continuous or ordinal outcomes under the competing risks scenario, which provide useful tools to validate and evaluate new joint modelling methods.
\end{abstract}

\section{Introduction}

Joint modeling of longitudinal and survival data has drawn a lot of attention over the past two decades. Much of the research has been focused on data with a single event time and a single type of failure, usually under the assumption of independent censoring of event times (Tsiatis and Davidian, 2004). However, in some situations interest lies with competing risks data, where there is more than one possible cause of an event or where the censoring is informative (Williamson et al., 2008). Typically, a standard linear mixed model or its extensions are used for the longitudinal submodel. Cause-specific hazards model with either unspecified or spline baseline hazards are studied for the competing risk submodels. Various types of random effects are assumed to account for the association between these submodels.

Despite various theoretical and methodological developments (Hickey et al., 2018b; Papageorgiou et al., 2019), there are still limited software packages to deal with specific problems in the analysis of follow-up data in clinical studies. To our knowledge, currently, there are three related CRAN R packages, namely JM (Rizopoulos, 2012), joineR (Williamson et al., 2008), and lcmm (Proust-Lima et al., 2017), which support the modeling of longitudinal and survival data with competing risks.

The JM package provides support for competing risks via the "CompRisk" option in the jointModel() function. In JM, a linear mixed-effects submodel is modeled for the longitudinal part and a relative risk submodel is assumed for each competing event. In the current version (1.4-8), only the piecewise proportional hazards model, where the log baseline hazard is approximated using B-splines, is supported for the survival component. The joineR package fits the joint model (Williamson et al., 2008) for joint models of longitudinal data and competing risks using the joint () function. In their model, the time-to-event data is modeled using a cause-specific Cox proportional hazards regression model with time-varying covariates. The longitudinal outcome is modeled using a linear mixed effects model. The association is captured by a zero-mean shared latent Gaussian process. Parameters in the model are estimated using an Expectation Maximization(EM) algorithm. The lcmm package implements the support for competing risks joint modeling in the Jointlcmm() function. Radically different from the above two R packages, the lcmm package uses a less well-known framework called the joint latent class model (Proust-Lima et al., 2014), which assumes that dependency between the longitudinal markers and the survival risk can be captured by a latent class structure entirely. However, the lcmm package is mainly designed for prediction purpose and may not be suited to evaluate specific assumptions regarding the characteristics of the marker trajectory that are the most influential on the event risk (Proust-Lima et al., 2014).

In all these packages, a time-independent shared random effects vector is usually assumed in modeling the longitudinal and survival data. However, they are not capable of fitting more flexible models with separate random effects in these submodels (Elashoff et al., 2008; Li et al., 2010). In many biomedical applications, sometimes, it is necessary to have a model which takes into account longitudinal ordinal outcomes for the longitudinal part. Yet, due to the complex nature of joint modeling, most of the available software does not support longitudinal ordinal variables (Armero et al., 2016; Ferrer, 2017). We thus decided to fill this gap and implemented a joint model which supports ordinal disease markers based on our previous work (Li et al., 2010).

Both JM and joineR packages depend heavily on the R nlme and survival packages. In JM, the linear mixed-effects submodel and the survival submodel are first fitted using lme() and coxph() $\mathrm{R}$ function in these packages before a joint modeling process. In joineR, Ime() and $\operatorname{coxph}()$ functions 
are applied to obtain initial values for parameters in the joint model, which are further estimated by an EM algorithm. The major advantage of using available packages such as survival and nlme lies that joint modeling $R$ packages can be built quickly with adequate efficiency as most of these base $R$ packages have been optimized for speed. However, if required functionality is not available in these packages, as is the case of Elashoff et al. (2008) and Li et al. (2010), implementing new joint modeling methods is a non-trivial task.

Compared with JM and joineR packages, the JMcmprsk package introduced here can be regarded as a "stand-alone" $\mathrm{R}$ package, which does not required initial estimates for the linear mixed effects model or survival submodel to compute parameters of the joint model in question. In particular, the JMcmprsk package is built within the Rcpp (Eddelbuettel et al., 2011) and GSL(The GNU Scientific Library)(Galassi et al., 2002) framework, which make R functions have access to a wide range of fast numerical routines such as Monte Carlo integration, numerical integration and differentiation.

\section{Joint Models with Competing Risks}

A joint model for competing risk data consists of two linked components: the longitudinal submodel, which takes care of repeatedly measured information and the survival submodel, which deals with multiple failure times. The combination of different longitudinal and survival components leads to a variety of joint models (Hickey et al., 2018a).

In the current version of JMcmprsk, we have implemented two joint models for competing risk data, namely joint modeling with continuous longitudinal outcomes (Elashoff et al., 2008), and joint modeling with ordinal longitudinal outcomes (Li et al., 2010). Both models have adopted a causespecific Cox submodel with a frailty term for multiple survival endpoints. The difference between these two models lies in the longitudinal part. The former model applies a linear mixed submodel for the continuous longitudinal outcome, while the latter model includes a partial proportional odds submodel for the ordinal longitudinal outcome.

Different from previous approaches (Rizopoulos, 2012; Williamson et al., 2008), we assume a flexible separate random effects structure for the longitudinal submodel and the survival submodel. Furthermore, the association between both submodels is modeled by the assumption that the random effects in two submodels jointly have a multivariate normal distribution.

\section{Model 1: Joint modeling with continuous longitudinal outcomes}

Let $Y_{i}(t)$ be the longitudinal outcome measured at time $t$ for subject $i, i=1,2, \cdots, n$ and $n$ is the total number of subjects in study. Let $C_{i}=\left(T_{i}, D_{i}\right)$ denote the competing risks data on subject $i$, where $T_{i}$ is the failure time or censoring time, and $D_{i}$ takes value in $\{0,1, \cdots, g\}$, with $D_{i}=0$ indicating a censored event and $D_{i}=k$ showing that subject $i$ fails from the $k$ th type of failure, where $k=1, \cdots, g$.

The joint model is specified in terms of the following two linked submodels:

$$
\begin{aligned}
Y_{i}(t) & =X_{i}^{(1)}(t)^{\top} \beta+\tilde{X}_{i}^{(1)}(t)^{\top} b_{i}+\epsilon_{i}(t), \\
\lambda_{k}(t) & =\lambda_{0 k}(t) \exp \left(X_{i}^{(2)}(t)^{\top} \gamma_{k}+v_{k} u_{i}\right), \text { for } k=1, \cdots, g,
\end{aligned}
$$

where $X_{i}^{(1)}(t), X_{i}^{(2)}(t)$ denote the covariates for the fixed-effects $\beta$ and $\gamma_{k}, \tilde{X}_{i}^{(1)}(t)$ denotes the covariates for the random-effects $b_{i}$ and $\epsilon_{i}(t) \sim N\left(0, \sigma^{2}\right)$ for all $t \geq 0$. The parameter $v_{1}$ is set to 1 to ensure identifiability. We assume that $b_{i}$ is independent of $\epsilon_{i}(t)$ and that $\epsilon_{i}\left(t_{1}\right)$ is independent of $\epsilon_{i}\left(t_{2}\right)$ for any $t_{1} \neq t_{2}$. We further assume the random effects $b_{i}$ and $u_{i}$ jointly have a multivariate normal distribution, denoted by $\theta_{i} \sim N(0, \Sigma)$, where $\Sigma=\left(\Sigma_{b}, \Sigma_{b u}^{\top} ; \Sigma_{b u}, \sigma_{u}\right)$.

Denote $\Psi$ as the unknown parameters from the joint models. We propose to obtain the maximum likelihood estimate of $\Psi$ through an EM algorithm. The complete data likelihood is

$$
\begin{aligned}
& L(\Psi ; Y, C, \theta) \\
& \propto \prod_{i=1}^{n}\left[\Pi_{j=1}^{n_{i}} \frac{1}{\sqrt{2 \pi \sigma^{2}}} \exp \left(-\frac{1}{2 \sigma^{2}}\left(Y_{i j}-X_{i}^{(1)}\left(t_{i j}\right)^{\top} \beta-\tilde{X}_{i}^{(1)}\left(t_{i j}\right)^{\top} b_{i}\right)^{2}\right)\right] \\
& \times \prod_{k=1}^{g} \lambda_{k}\left(T_{i}\right)^{I\left(D_{i}=k\right)} \exp \left\{-\int_{0}^{T_{i}} \sum_{k=1}^{g} \lambda_{k}(t) d t\right\} \\
& \times \frac{1}{\sqrt{(2 \pi)^{d}|\Sigma|}} \exp \left(-\frac{1}{2} \theta_{i}^{\top} \Sigma^{-1} \theta_{i}\right) .
\end{aligned}
$$


In the E-step, we need to calculate the expected value of all the functions of $\theta$. Since the integral over the random effects does not have a closed-form solution, an iterative numerical method has to be employed.

In JMcmprsk, the integral over time is approximated using a Gauss-Kronrod quadrature and the computation of the integral over the individual random effects is achieved using a Gauss-Hermite quadrature. The quadrature approximates the integral using a weighted sum of function values at specified points within the domain of integration; the Gaussian quadrature is based on the use of polynomial functions. A standard option here is the Gaussian quadratic rules. In the $M-s t e p, \Psi$ is updated by maximizing the functions obtained from the E-step.

\section{Model 2: Joint modeling with ordinal longitudinal outcomes}

Let $Y_{i j}$ denote the $j$ th response measured on subject $i$, where $i=1, \cdots, n, j=1, \cdots, n_{i}$, and $Y_{i j}$ takes values in $\{1, \cdots, K\}$. The competing risks failure times on subject $i$ is $\left(T_{i}, D_{i}\right)$, and the notations have the same meaning as in Model 1.

We propose the following partial proportional odds model for $Y_{i j}$

$$
P\left(Y_{i j} \leq k \mid X_{i j}, \tilde{X}_{i j}, W_{i j}, b_{i}\right)=\frac{1}{1+\exp \left(-\theta_{k}-X_{i j} \beta-\tilde{X}_{i j} \alpha_{k}-W_{i j}^{\top} b_{i}\right)},
$$

where $k=1, \cdots, K-1, X_{i j}$ and $\tilde{X}_{i j}$ are $p \times 1$ and $s \times 1$ vectors of covariates for the fixed-effect $\beta$ and $\alpha_{k}$, with $\alpha_{1}=0$, and $\tilde{X}_{i j}$ is a subset of $X_{i j}$ for which the proportional odds assumption may not be satisfied. The $q \times 1$ vector $b_{i}$ represents random effects of the associated covariates $W_{i j}$.

The distribution of the competing risks failure times $\left(T_{i}, D_{i}\right)$ are assumed to take the form of the following cause-specific hazards frailty model:

$$
\lambda_{d}\left(t \mid Z_{i}(t), u_{i}\right)=\lambda_{0 d}(t) \exp \left(Z_{i}(t)^{\top} \gamma_{d}+v_{d} u_{i}\right), \text { for } d=1, \cdots, g,
$$

where the $l \times 1$ vector $\gamma_{d}$ and $v_{d}$ are the cause-specific coefficients for the covariates $Z_{i}(t)$ and the random effects $u_{i}$, respectively.

The parameter $v_{1}$ is set to 1 to ensure identifiability. We assume the random effects $b_{i}$ and $u_{i}$ jointly have a multivariate normal distribution, denoted by $a_{i} \sim N(0, \Sigma)$.

Denote $\Psi$ as the unknown parameters from the joint models. We propose to obtain the maximum likelihood estimate of $\Psi$ through an EM algorithm. The complete data likelihood is

$$
\begin{aligned}
& L(\Psi ; Y, C, a) \\
& \propto \prod_{i=1}^{n}\left[\Pi_{j=1}^{n_{i}} \Pi_{k=1}^{K}\left\{\pi_{i j}(k)-\pi_{i j}(k-1)\right\}^{I\left(Y_{i j}=k\right)}\right] \\
& \times \Pi_{d=1}^{g} \lambda_{d}\left(T_{i}\right)^{I\left(D_{i}=d\right)} \exp \left\{-\int_{0}^{T_{i}} \sum_{k=1}^{d} \lambda_{d}(t) d t\right\} \\
& \times \frac{1}{\sqrt{(2 \pi)^{q+1}|\Sigma|}} \exp \left(-\frac{1}{2} a_{i}^{\top} \Sigma^{-1} a_{i}\right) .
\end{aligned}
$$

where $\pi_{i j}(k)$ stands for the probability that $Y_{i j} \leq k$ given the covariates and the random effects. The implementation of EM algorithm in this case is similar to the procedure of Model 1.

\section{Package structure and functionality}

The R package JMcmprsk implements the above two joint models on the basis of R package Rcpp (Eddelbuettel et al., 2011) and GSL library(Galassi et al., 2002) and is hosted at CRAN. After setting the GSL environment by following the instructions in the INSTALL file from the package, we can issue the following command in the R console to install the package:

> install.packages("JMcmprsk")

There are two major functions included in the JMcmprsk package: the function that fits continuous outcomes jmc() and the function that fits ordinal outcomes jmo(). 


\section{jmc() function}

As an illustrative example of jmc(), we consider Scleroderma Lung Study (Tashkin et al., 2006), a double-blinded, randomized clinical trial to evaluate the effectiveness of oral cyclophosphamide (CYC) versus placebo in the treatment of lung disease due to scleroderma. This study consists of 158 patients and the primary outcome is forced vital capacity (FVC, as \% predicted) determined at 3-month intervals from the baseline. The event of interest is the time-to-treatment failure or death. We consider two covariates, baseline \%FVC $\left(F V C_{0}\right)$ and baseline lung fibrosis $\left(F I B_{0}\right)$ and two risks, informative and noninformative. The model setups are as follows:

$$
\begin{aligned}
\% F V C_{i j}= & \beta_{0}+\beta_{1} t_{i j}+\beta_{2} F V C_{0 i}+\beta_{3} F I B_{0 i}+\beta_{4} C Y C_{i} \\
& +\beta_{5} F V C_{0 i} \times C Y C_{i}+\beta_{6} F I B_{0 i} \times C Y C_{i}+\beta_{7} t_{i j} \times C Y C_{i}+b_{i} t_{i j}+\epsilon,
\end{aligned}
$$

and the cause-specific hazards frailty models are

$$
\begin{array}{r}
\lambda_{1}(t)=\lambda_{01}(t) \exp \left(\gamma_{11} F V C_{0 i}+\gamma_{12} F I B_{0 i}+\gamma_{13} C Y C_{i}+\gamma_{14} F V C_{0 i} \times C Y C_{i}+\gamma_{15} F I B_{0 i} \times C Y C_{i}+u_{i}\right) \\
\lambda_{2}(t)=\lambda_{02}(t) \exp \left(\gamma_{21} F V C_{0 i}+\gamma_{22} F I B_{0 i}+\gamma_{23} C Y C_{i}+\gamma_{24} F V C_{0 i} \times C Y C_{i}+\gamma_{25} F I B_{0 i} \times C Y C_{i}+v_{2} u_{i}\right),
\end{array}
$$

We first load the package and the data.

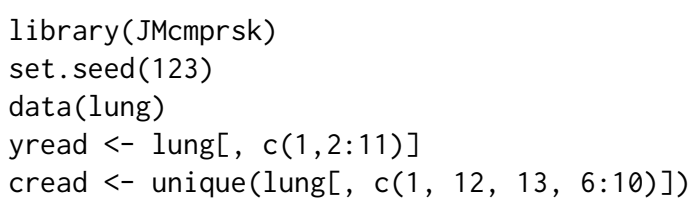

The number of rows in "yread" is the total number of measurements for all subjects in the study. For "cread", the survival/censoring time is included in the first column, and the failure type coded as 0 (censored events), 1 (risk 1), or 2 (risk 2 ) is given in the second column. Two competing risks are assumed.

Then, "yread" and "cread" are used as the longitudinal and survival input data for the model specified by the function $\mathrm{jmc}()$ as shown below:

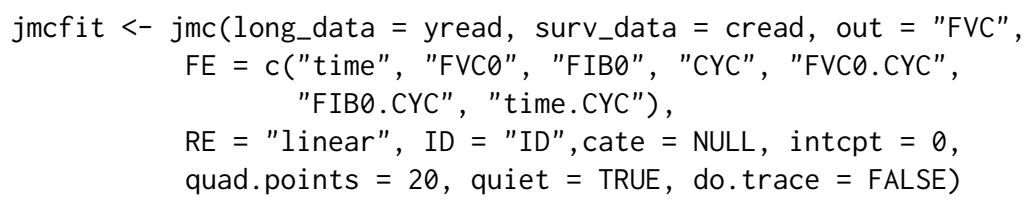

where out is the name of the outcome variable in the longitudinal sub-model, FE the list of covariates for the fixed effects in the longitudinal sub-model, RE the types/vector of random effects in the longitudinal sub-model, ID the column name of subject id, cate the list of categorical variables for the fixed effects in the longitudinal sub-model, intcpt the indicator of random intercept coded as 1 (yes, default) or 0(no). The option quiet is used to print the progress of function, the default is TRUE (no printing).

A concise summary of the results can be obtained using jmcfit as shown below:

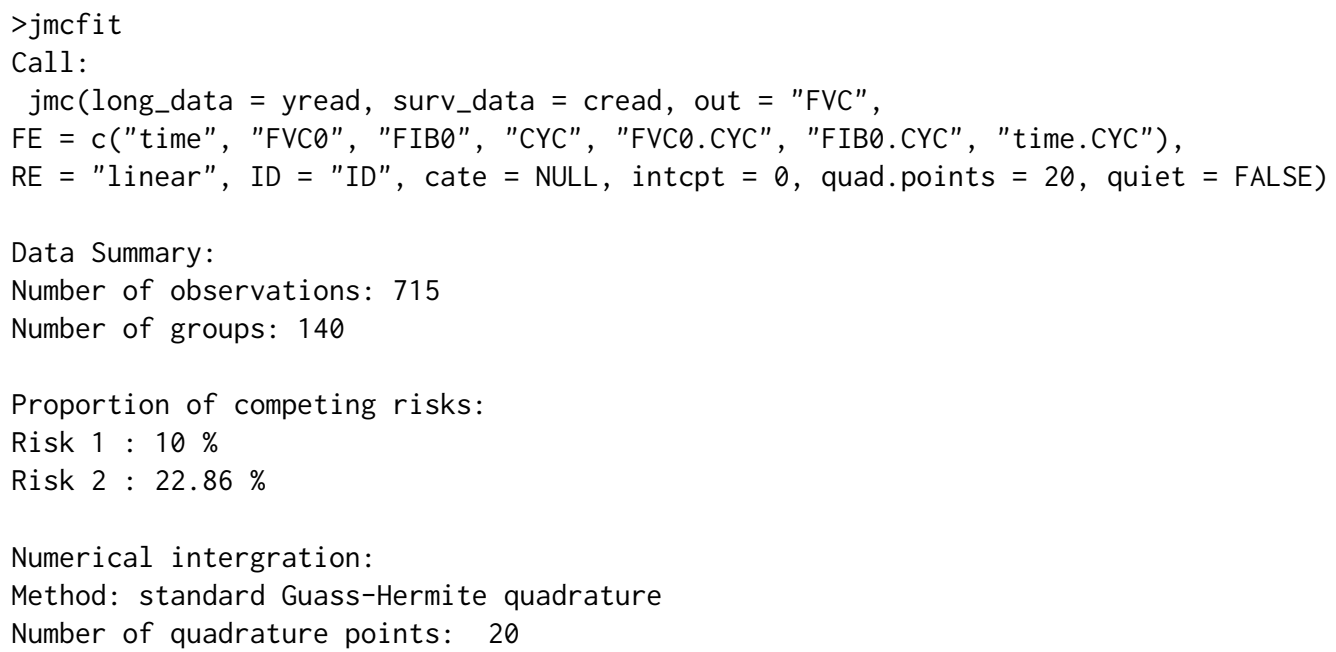


Model summary:

Longitudinal process: linear mixed effects model

Event process: cause-specific Cox proportional hazard model with unspecified baseline hazard

Loglikelihood: $\quad-3799.044$

Longitudinal sub-model fixed effects: FVC time + FVCO + FIBO + CYC + FVCO.CYC + FIBO.CYC + time.CYC

\begin{tabular}{lllcr}
\multicolumn{1}{c}{ Estimate } & Std. Error & $95 \%$ CI & $\operatorname{Pr}(>|Z|)$ \\
$\begin{array}{c}\text { Longitudinal: } \\
\text { Fixed effects: }\end{array}$ & & & & \\
intercept & 66.0415 & 0.7541 & $(64.5634,67.5196)$ & 0.0000 \\
time & -0.0616 & 0.0790 & $(-0.2165,0.0932)$ & 0.4353 \\
FVC0 & 0.9017 & 0.0365 & $(0.8302,0.9732)$ & 0.0000 \\
FIB0 & -1.7780 & 0.5605 & $(-2.8767,-0.6793)$ & 0.0015 \\
CYC & 0.0150 & 0.9678 & $(-1.8819,1.9119)$ & 0.9876 \\
FVC0.CYC & 0.1380 & 0.0650 & $(0.0106,0.2654)$ & 0.0338 \\
FIB0.CYC & 1.7088 & 0.7643 & $(0.2109,3.2068)$ & 0.0254 \\
time.CYC & 0.1278 & 0.1102 & $(-0.0883,0.3438)$ & 0.2464 \\
Random effects: & & & & \\
sigma^2 & 22.7366 & 0.6575 & $(21.4478,24.0253)$ & 0.0000
\end{tabular}

Survival sub-model fixed effects: Surv(surv, failure_type) $\sim$ FVCO + FIB $0+$ CYC + FVCO.CYC + FIB 0. CYC

$\begin{array}{lllll} & \text { Estimate } & \text { Std. Error } & 95 \% \text { CI } & \operatorname{Pr}(>|Z|) \\ \text { Survival: } & & & & \\ \text { Fixed effects: } & & & & \\ \text { FVC0_1 } & 0.0187 & 0.0326 & (-0.0452,0.0826) & 0.5660 \\ \text { FIB0_1 } & 0.1803 & 0.3521 & (-0.5098,0.8705) & 0.6086 \\ \text { CYC_1 } & -0.6872 & 0.7653 & (-2.1872,0.8128) & 0.3692 \\ \text { FVC0.CYC_1 } & -0.0517 & 0.0746 & (-0.1979,0.0945) & 0.4880 \\ \text { FIB0.CYC_1 } & -0.4665 & 1.1099 & (-2.6419,1.7089) & 0.6743 \\ \text { FVC0_2 } & -0.0677 & 0.0271 & (-0.1208,-0.0147) & 0.0123 \\ \text { FIB0_2 } & 0.1965 & 0.3290 & (-0.4484,0.8414) & 0.5503 \\ \text { CYC_2 } & 0.3137 & 0.4665 & (-0.6007,1.2280) & 0.5013 \\ \text { FVC0.CYC_2 } & 0.1051 & 0.0410 & (0.0248,0.1854) & 0.0103 \\ \text { FIB0.CYC_2 } & 0.1239 & 0.4120 & (-0.6836,0.9314) & 0.7636 \\ & & & & \\ \text { Association parameter: } & & & & \\ \text { V2 } & 1.9949 & 2.3093 & (-2.5314,0.5212) & 0.3877 \\ \text { Random effects: } & & & & \\ \text { sigma_b11 } & 0.2215 & 0.0294 & (0.1638,0.2792) & 0.0000 \\ \text { sigma_u } & 0.0501 & 0.0898 & (-0.1259,0.2260) & 0.5772 \\ \text { Covariance: } & & & & \\ \text { sigma_b1u } & -0.0997 & 0.0797 & (-0.2560,0.0565) & 0.2109\end{array}$

The resulting table contains three parts, the fixed effects in longitudinal model, survival model and random effects. It gives the estimated parameters in the first column, the standard error in the second column, and $95 \%$ confidence interval and $p$-value for these parameters in the third and fourth columns. In our example, there is only one random effect. If there is more than one random effect, the output will include $\operatorname{sigma}_{b} 11$, $\operatorname{sigma}_{b} 12$, $\operatorname{sigma}_{b} 22$, sigma 1 1u, sigma $2 u$, and so on.

The supporting function coef () can be used to extract the coefficients of the longitudinal/survival process by specifying the argument coeff, where"beta" and "gamma" denotes the longitudinal and survival submodel fixed effects, respectively.

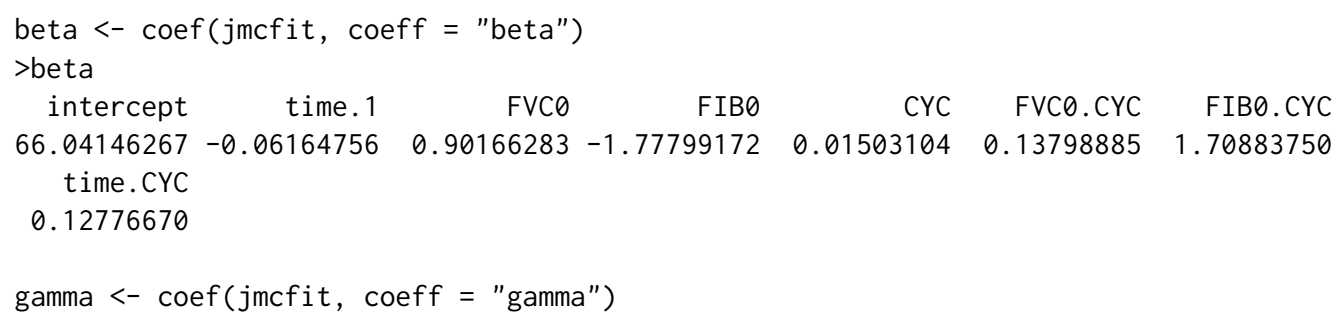




$\begin{array}{lrrrrr}\text { >gamma } & & & & & \\ & \text { FVC0 } & \text { FIB0 } & \text { CYC } & \text { FVC0.CYC } & \text { FIB0.CYC } \\ {[1,]} & 0.01871359 & 0.1803249 & -0.6872099 & -0.05172157 & -0.4664724 \\ {[2,]} & -0.06772664 & 0.1965190 & 0.3136709 & 0.10509986 & 0.1239203\end{array}$

The supporting function summary () can be used to extract the point estimate, the standard error, $95 \% \mathrm{CI}$, and $p$-values of the coefficients of both sub-models with the option coeff to specify which submodel fixed effects one would like to extract, and digits, the number of digits to be printed out. We proceed below to extract the fixed effects for both submodels:

\begin{tabular}{|c|c|c|c|c|c|c|c|}
\hline \multicolumn{2}{|c|}{ Longitudinal } & \multicolumn{5}{|c|}{ SE 95\%Lower 95\%Upper p-values } & \\
\hline 1 & intercept & 66.0415 & 0.7541 & 64.5634 & 67.5196 & 0.0000 & \\
\hline 2 & time & -0.0616 & 0.0790 & -0.2165 & 0.0932 & 0.4353 & \\
\hline 3 & FVCO & 0.9017 & 0.0365 & 0.8302 & 0.9732 & 0.0000 & \\
\hline 4 & FIBO & -1.7780 & 0.5605 & -2.8767 & -0.6793 & 0.0015 & \\
\hline & $\mathrm{CYC}$ & 0.0150 & 0.9678 & -1.8819 & 1.9119 & 0.9876 & \\
\hline 6 & FVCO.CYC & 0.1380 & 0.0650 & 0.0106 & 0.2654 & 0.0338 & \\
\hline 7 & FIBO.CYC & 1.7088 & 0.7643 & 0.2109 & 3.2068 & 0.0254 & \\
\hline 8 & time.CYC & 0.1278 & 0.1102 & -0.0883 & 0.3438 & 0.2464 & \\
\hline \multicolumn{8}{|c|}{ >summary (jmcfit, coeff = "survival", digits = 4) } \\
\hline & Survival & coef ex & $x p(c o e f)$ & SE (coef) & 95\%Lower & 95\%Upper & \\
\hline 1 & FVC0_1 & 0.0187 & 1.0189 & 0.0326 & -0.0452 & 0.0826 & 660 \\
\hline 2 & FIBO_1 & 0.1803 & 1.1976 & 0.3521 & -0.5098 & 0.8705 & 0.6086 \\
\hline 3 & CYC_1 & -0.6872 & 0.5030 & 0.7653 & -2.1872 & 0.8128 & 0.3692 \\
\hline 4 & FVCO.CYC_1 & -0.0517 & 0.9496 & 0.0746 & -0.1979 & 0.0945 & 0.4880 \\
\hline 5 & FIBO.CYC_1 & -0.4665 & 0.6272 & 1.1099 & -2.6419 & 1.7089 & 0.6743 \\
\hline 6 & FVCO_2 & -0.0677 & 0.9345 & 0.0271 & -0.1208 & -0.0147 & 0.0123 \\
\hline 7 & FIB0_2 & 0.1965 & 1.2172 & 0.3290 & -0.4484 & 0.8414 & 0.5503 \\
\hline 8 & CYC_2 & 0.3137 & 1.3684 & 0.4665 & -0.6007 & 1.2280 & 0.5013 \\
\hline & FVCO.CYC_2 & 0.1051 & 1.1108 & 0.0410 & 0.0248 & 0.1854 & 0.0103 \\
\hline 10 & FIBO.CYC_2 & 0.1239 & 1.1319 & 0.4120 & -0.6836 & 0.9314 & 0.7636 \\
\hline
\end{tabular}

We proceed to test the global hypothesis for the longitudinal and the survival submodels using linearTest().

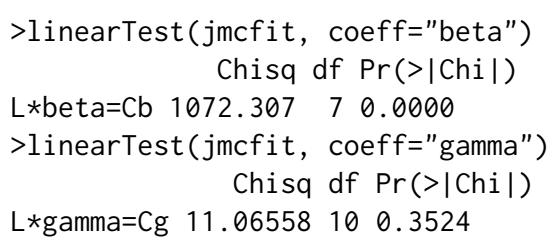

The results suggest that the hypothesis $\beta_{1}=\beta_{2}=\cdots=\beta_{7}=0$ is rejected, and the hypothesis $\gamma_{11}=\gamma_{12}=\cdots=\gamma_{15}=\gamma_{21}=\gamma_{22}=\cdots=\gamma_{25}=0$ is not rejected at the significance level of 0.05 .

linearTest () can also be used to test any linear hypothesis about the coefficients for each submodel. For example, if one wants to test $H_{0}: \beta_{1}=\beta_{2}$ in the longitudinal submodel, then we start with a linear contrast Lb and pass it to linearTest().

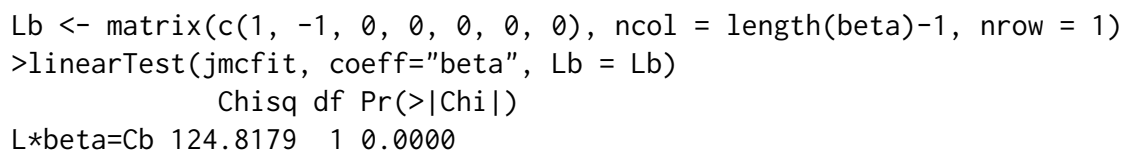

Note that we do not include intercept for linear hypotheses testing. It is seen that the hypothesis $\beta_{1}=\beta_{2}$ is rejected at level 0.05 in the above example.

Similarly, a linear hypotheses testing can also be done in the survival submodel using linearTest (). For example, if we want to test $H_{0}: \gamma_{11}=\gamma_{21}$, then we start with another linear contrast Lg and pass it to linearTest().

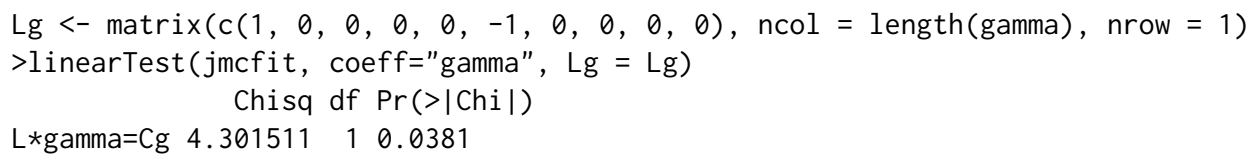


It is seen that the hypothesis $\gamma_{11}=\gamma_{21}$ is rejected at level 0.05 .

For categorical variables, jmc () function will create the appropriate dummy variables automatically as needed within the function. The reference group in a categorical variable is specified as the one that comes first alphabetically. Below is another example:

First, we add two categorical variables "sex" and "race" to the longitudinal data set "yread", in which "sex" is coded as "Female" or "Male", and race is coded as "Asian", "White", "Black", or "Hispanic".

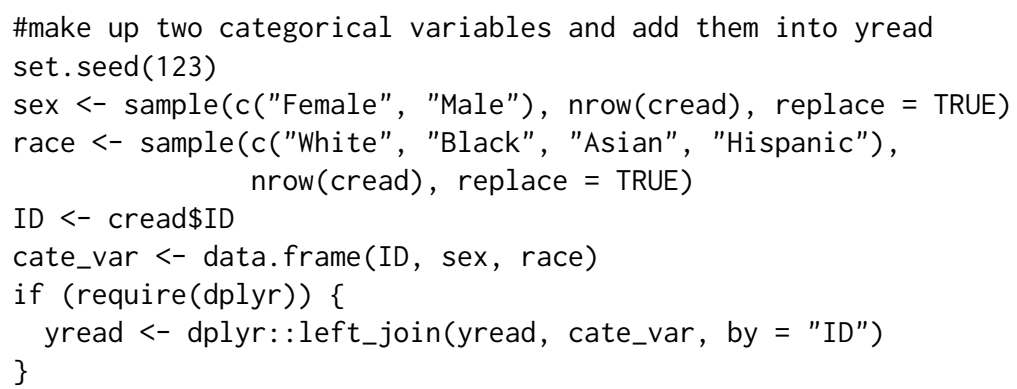

Second, we rerun the model with the two added categorical variables.

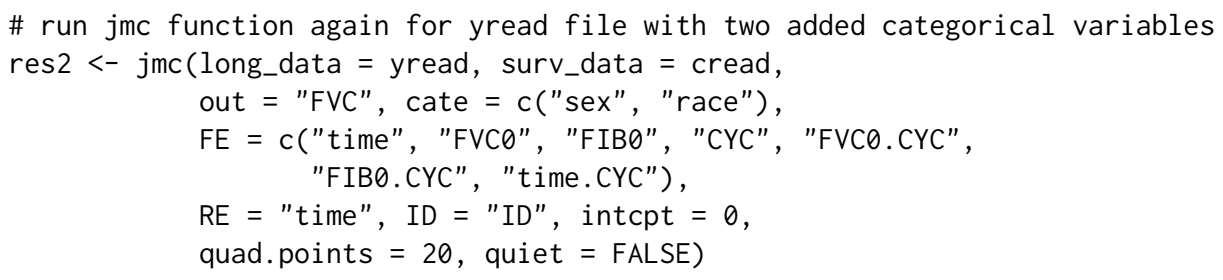

We can obtain the estimated coefficients of the longitudinal process using coef().

\begin{tabular}{|c|c|c|c|c|c|c|c|}
\hline intercept & time & FVCO & FIBO & CYC & FVCO.CYC & FIBO.CYC & time.CYC \\
\hline 67.05760799 & -0.07340060 & 0.91105151 & -1.75007966 & 0.02269507 & 0.13045588 & 1.58807248 & 0.15876200 \\
\hline Male & Black & Hispanic & White & & & & \\
\hline 0.77110697 & -0.94635182 & -0.45873814 & -1.19910638 & & & & \\
\hline
\end{tabular}

\section{jmo() function}

The implementation of $j \mathrm{mo}()$ is very similar to that of $j \mathrm{mc}()$. As an illustrative example, we use the data from (rt PA Stroke Study, 1995). In this study, 624 patients are included, and the patients treated with rt-PA were compared with those given placebo to look for an improvement from baseline in the score on the modified Rankin scale, an ordinal measure of the degree of disability with categories ranging from no symptoms, no significant disability to severe disability or death, which means in this example, $Y_{i j}$ takes $K=4$ ordinal values. The following covariates are considered: treatment group (rt-PA or placebo), modified Rankin scale prior stroke onset, time since randomization (dummy variables for 3, 6 and 12 months), and the three subtypes of acute stroke (small vessel occlusive disease, large vessel atherosclerosis or cardioembolic stroke, and unknown reasons). Similarly, we also consider the informative and noninformative risks. The model setups are as follows:

$$
\begin{aligned}
P\left(Y_{i j} \leq k\right)= & {\left[1+\exp \left(-\theta_{k}-\left(\beta_{1} \text { Group }+\beta_{2} \text { Modified Rankin scale prior onset }+\beta_{3} \text { time } 3\right.\right.\right.} \\
& +\beta_{4} \text { time6 }+\beta_{5} \text { time } 12+\beta_{6} \text { Small vessel }+\beta_{7} \text { Large vessel or cardioembolic stroke } \\
& \left.+\beta_{8} \text { Small vessel }{ }^{*} \text { group }+\beta_{9} \text { Large vessel or cardioembolic stroke }{ }^{*} \text { group }\right) \\
& \left.\left.-\left(\alpha_{k 1} \text { Small vessel }+\alpha_{k 2} \text { Large vessel or cardioembolic stroke }\right)-b_{i}\right)\right]^{-1}
\end{aligned}
$$

where $k=1, \cdots, K-1$.

$$
\begin{aligned}
& \lambda_{1}(t)=\lambda_{01}(t) \exp \left(\gamma_{11} \text { Group }+\gamma_{12}\right. \text { Modified Rankin scale prior onset } \\
& +\gamma_{13} \text { Small vessel }+\gamma_{14} \text { Large vessel or cardioembolic stroke } \\
& +\gamma_{15} \text { Small vessel }{ }^{*} \text { group }+\gamma_{16} \text { Large vessel or cardioembolic stroke* group }+u_{i} \text { ) } \\
& \lambda_{2}(t)=\lambda_{02}(t) \exp \left(\gamma_{21} \text { Group }+\gamma_{22}\right. \text { Modified Rankin scale prior onset } \\
& +\gamma_{23} \text { Small vessel }+\gamma_{24} \text { Large vessel or cardioembolic stroke } \\
& +\gamma_{25} \text { Small vessel }{ }^{*} \text { group }+\gamma_{26} \text { Large vessel or cardioembolic stroke* group }+v_{2} u_{i} \text { ) }
\end{aligned}
$$


We first load the package and the data.

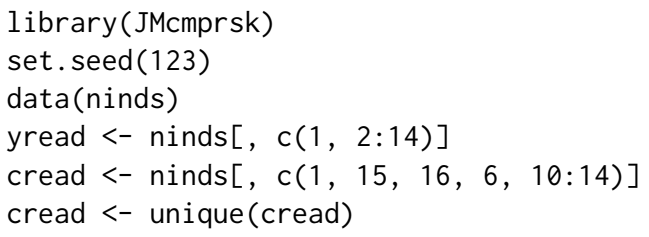

and the other arrangements are the same with those in $\operatorname{jmc}()$,

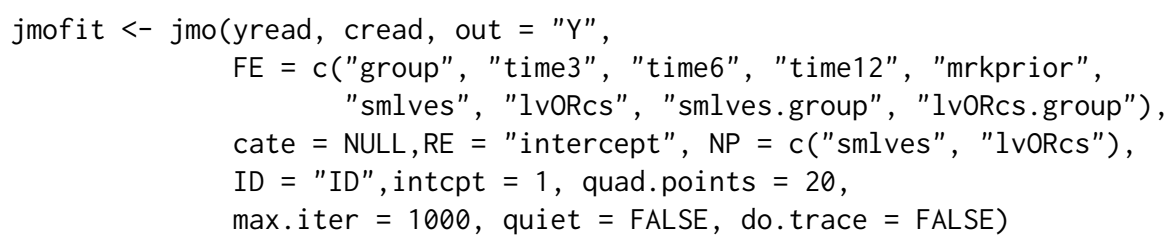

where NP is the list of non-proportional odds covariates and FE the list of proportional odds covariates.

To see a concise summary of the result, we can type:

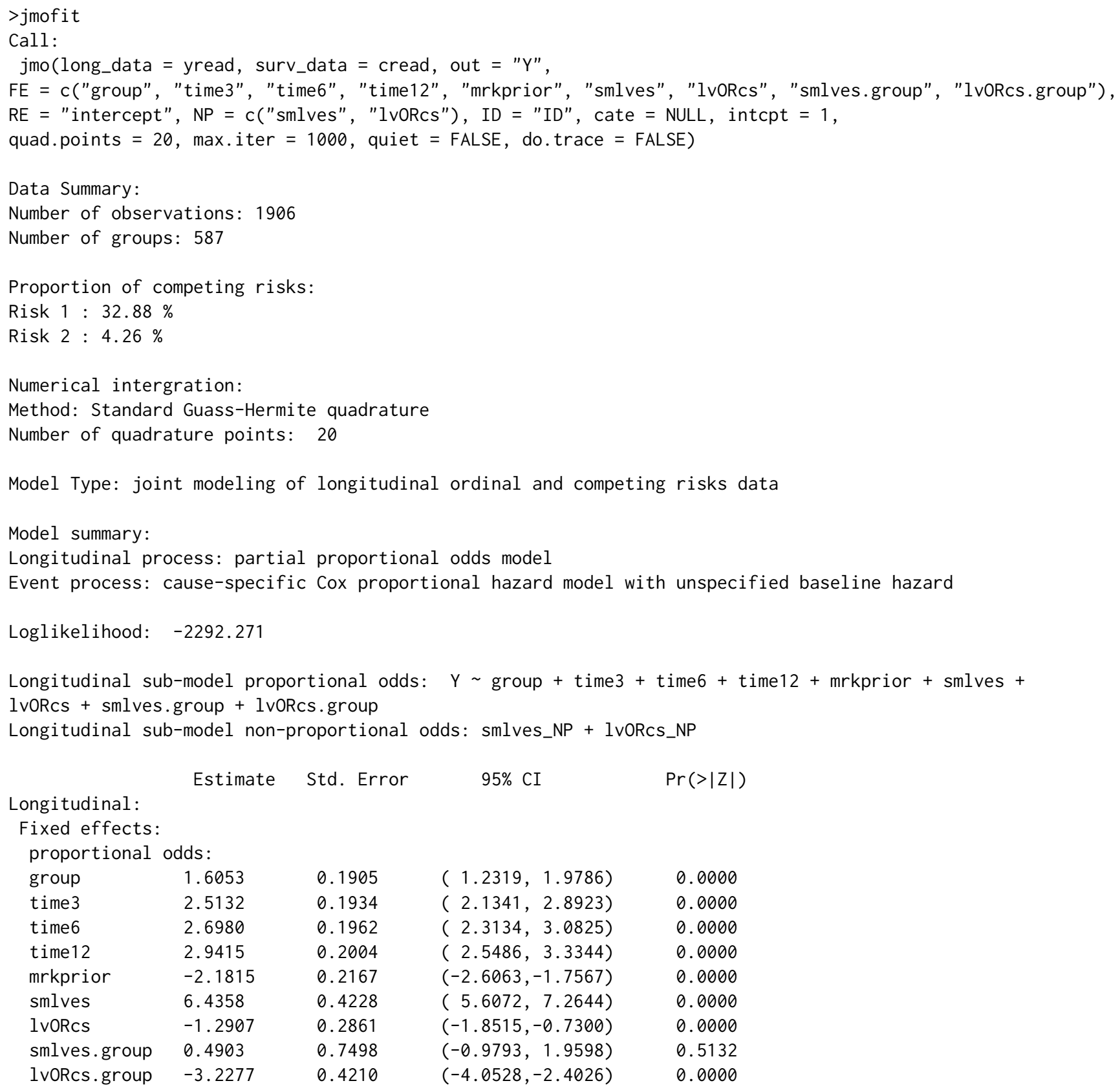

$\begin{array}{lllll}\text { group } & 1.6053 & 0.1905 & (1.2319,1.9786) & 0.0000 \\ \text { time3 } & 2.5132 & 0.1934 & (2.1341,2.8923) & 0.0000 \\ \text { time6 } & 2.6980 & 0.1962 & (2.3134,3.0825) & 0.0000 \\ \text { time12 } & 2.9415 & 0.2004 & (2.5486,3.3344) & 0.0000 \\ \text { mrkprior } & -2.1815 & 0.2167 & (-2.6063,-1.7567) & 0.0000 \\ \text { smlves } & 6.4358 & 0.4228 & (5.6072,7.2644) & 0.0000 \\ \text { lvORcs } & -1.2907 & 0.2861 & (-1.8515,-0.7300) & 0.0000 \\ \text { smlves.group } & 0.4903 & 0.7498 & (-0.9793,1.9598) & 0.5132 \\ \text { lvORcs.group } & -3.2277 & 0.4210 & (-4.0528,-2.4026) & 0.0000\end{array}$


Non-proportional odds:

$\begin{array}{lllll}\text { smlves_NP_2 } & 0.2725 & 0.4485 & (-0.6066,1.1515) & 0.5435 \\ \text { lvORcs_NP_2 } & -0.4528 & 0.2466 & (-0.9362,0.0305) & 0.0663 \\ \text { smlves_NP_3 } & 1.7844 & 1.0613 & (-0.2958,3.8645) & 0.0927 \\ \text { lvORcs_NP_3 } & -0.1364 & 0.4309 & (-0.9809,0.7081) & 0.7516 \\ \text { Logit-specific } & \text { intercepts: } & & & \\ \text { theta1 } & -6.2336 & 0.1722 & (-6.5712,-5.8960) & 0.0000 \\ \text { theta2 } & -4.1911 & 0.1561 & (-4.4971,-3.8851) & 0.0000 \\ \text { theta3 } & 3.9806 & 0.1896 & (3.6091,4.3522) & 0.0000\end{array}$

Survival sub-model fixed effects: Surv(surv, comprisk) $\sim$ group + mrkprior + smlves + lvORcs + smlves.group + lvORcs.group

\begin{tabular}{|c|c|c|c|c|}
\hline & Estimate & Std. Error & $95 \% \mathrm{CI}$ & $\operatorname{Pr}(>|Z|)$ \\
\hline \multicolumn{5}{|l|}{ Survival: } \\
\hline \multicolumn{5}{|l|}{ Fixed effects: } \\
\hline group_1 & -0.4630 & 0.2434 & $(-0.9400,0.0140)$ & 0.0571 \\
\hline mrkprior_1 & 0.5874 & 0.1371 & $(0.3187,0.8560)$ & 0.0000 \\
\hline smlves_1 & -2.5570 & 0.7223 & $(-3.9728,-1.1413)$ & 0.0004 \\
\hline 1vORcs_1 & 0.5992 & 0.2485 & $(0.1120,1.0863)$ & 0.0159 \\
\hline smlves.group_1 & -0.4990 & 1.4257 & $(-3.2934,2.2955)$ & 0.7264 \\
\hline lvORcs.group_1 & 1.1675 & 0.4692 & $(0.2479,2.0871)$ & 0.0128 \\
\hline group_2 & 0.2087 & 0.4834 & $(-0.7388,1.1562)$ & 0.6659 \\
\hline mrkprior_2 & 0.0616 & 0.4277 & $(-0.7766,0.8998)$ & 0.8854 \\
\hline smlves_2 & 0.7758 & 0.6217 & $(-0.4428,1.9943)$ & 0.2121 \\
\hline lvORcs_2 & -0.3256 & 0.5120 & $(-1.3291,0.6778)$ & 0.5247 \\
\hline smlves.group_2 & -0.0437 & 1.1573 & $(-2.3120,2.2245)$ & 0.9699 \\
\hline lvORcs.group_2 & 0.0991 & 1.0718 & $(-2.0015,2.1998)$ & 0.9263 \\
\hline \multicolumn{5}{|c|}{ Association prameter: } \\
\hline v2 & 0.0101 & 0.1595 & $(-0.3025,0.3227)$ & 0.9496 \\
\hline \multicolumn{5}{|l|}{ Random effects: } \\
\hline sigma_b11 & 55.6404 & 5.6560 & $(44.5547,66.7261)$ & 0.0000 \\
\hline sigma_u & 6.6598 & 1.7196 & $(3.2894,10.0303)$ & 0.0001 \\
\hline \multicolumn{5}{|l|}{ Covariance: } \\
\hline sigma_b1u & -19.2452 & 0.7730 & $(-20.7602,-17.7302)$ & 0.0000 \\
\hline
\end{tabular}

The usage of function coef () is similar to those in Model 1. More specifically, coef() can extract the coefficients of non-proportional odds fixed effects and logit-specific intercepts. For example,

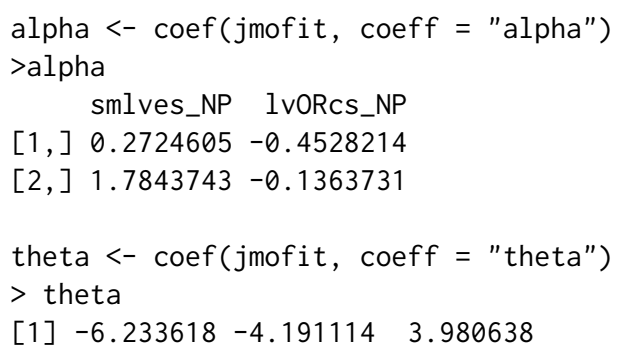

The usage of function summary () is the same as in Model 1. It extracts the point estimate, standard error, $95 \% \mathrm{CI}$, and $p$-values of the coefficients of both submodels as demonstrated below:

$\begin{array}{lrrrrrr}>\text { summary(jmofit, coeff }= & \text { "longitudinal") } & \\ & \text { Longitudinal } & \text { coef } & \text { SE } & 95 \% \text { Lower } & 95 \% \text { Upper } & \text { p-values } \\ 1 & \text { group } & 1.6053 & 0.1905 & 1.2319 & 1.9786 & 0.0000 \\ 2 & \text { time3 } & 2.5132 & 0.1934 & 2.1341 & 2.8923 & 0.0000 \\ 3 & \text { time6 } & 2.6980 & 0.1962 & 2.3134 & 3.0825 & 0.0000 \\ 4 & \text { time12 } & 2.9415 & 0.2004 & 2.5486 & 3.3344 & 0.0000 \\ 5 & \text { mrkprior } & -2.1815 & 0.2167 & -2.6063 & -1.7567 & 0.0000 \\ 6 & \text { smlves } & 6.4358 & 0.4228 & 5.6072 & 7.2644 & 0.0000 \\ 7 & \text { lvORcs } & -1.2907 & 0.2861 & -1.8515 & -0.7300 & 0.0000 \\ 8 & \text { smlves.group } & 0.4903 & 0.7498 & -0.9793 & 1.9598 & 0.5132 \\ 9 & \text { lvORcs.group } & -3.2277 & 0.4210 & -4.0528 & -2.4026 & 0.0000\end{array}$




\begin{tabular}{|c|c|c|c|c|c|c|c|c|c|}
\hline smlves_NP_2 & $0.2725 e$ & 0.4485 & -0.6 & 066 & 1.1 & 515 & 0.54 & 435 & \\
\hline 1vORcs_NP_2 - & $-0.4528 e$ & 0.2466 & -0.9 & 362 & 0.0 & 305 & 0.06 & 663 & \\
\hline smlves_NP_3 & 1.78441 & 1.0613 & -0.2 & 958 & 3.8 & 3645 & 0.09 & 927 & \\
\hline lvORcs_NP_3 - & $-0.1364 e$ & 0.4309 & -0.9 & 809 & 0.7 & 081 & 0.75 & 516 & \\
\hline theta1 & $-6.2336 e$ & 0.1722 & -6.5 & 712 & -5.8 & 3960 & 0.00 & 000 & \\
\hline theta2 - & $-4.1911 e$ & 0.1561 & -4.4 & 971 & -3.8 & 3851 & 0.00 & 000 & \\
\hline theta3 & $3.9806 e$ & 0.1896 & 3.6 & 091 & 4.3 & 3522 & 0.00 & 000 & \\
\hline mmary (jmofit, & $\therefore$ coeff $=$ & $=$ "sur & & & & & & & \\
\hline Survival & al coef & $f \exp (c$ & pef) & $\mathrm{SE}(\mathrm{co}$ & oef) & 95\% Lowe & ver 9 & 95\%Upper & p-values \\
\hline group_1 & $-1-0.463 e$ & & 6294 & & 2434 & -0.94 & & 0.0140 & 0.0571 \\
\hline mrkprior_1 & 10.5874 & & 7993 & & 1371 & $0.31 \varepsilon$ & 87 & 0.8560 & 0.0000 \\
\hline smlves_1 & $-1-2.557 e$ & & 8775 & & 7223 & -3.97 & 28 & -1.1413 & 0.0004 \\
\hline lvORcs_1 & 10.5992 & & 8206 & & 2485 & 0.112 & 20 & 1.0863 & 0.0159 \\
\hline smlves.group_1 & $1-0.499 e$ & & 6072 & & 4257 & -3.29 & 34 & 2.2955 & 0.7264 \\
\hline lvORcs.group_1 & 11.1675 & & 2140 & & 4692 & 0.24 & 479 & 2.0871 & 0.0128 \\
\hline group_2 & 0.2087 & & 2321 & & 4834 & $-0.73 \varepsilon$ & & 1.1562 & 0.6659 \\
\hline mrkprior_2 & 0.0616 & & 2636 & & 4277 & $-0.77 €$ & 766 & 0.8998 & 0.8854 \\
\hline smlves_2 & 20.7758 & & 1722 & & 6217 & -0.442 & 428 & 1.9943 & 0.2121 \\
\hline lvORcs_2 & $2-0.3256$ & & 7221 & & 5120 & -1.32 & 291 & 0.6778 & 0.5247 \\
\hline smlves.group_2 & $2-0.0437$ & & 9572 & & 1573 & -2.312 & 120 & 2.2245 & 0.9699 \\
\hline IvORcs.group_2 & 20.0991 & & 1042 & & 0718 & -2.00 & & 2.1998 & 0.9263 \\
\hline
\end{tabular}

Analogous to jmcfit, linearTest() can be used to the global hypothesis for the longitudinal and the survival submodels.

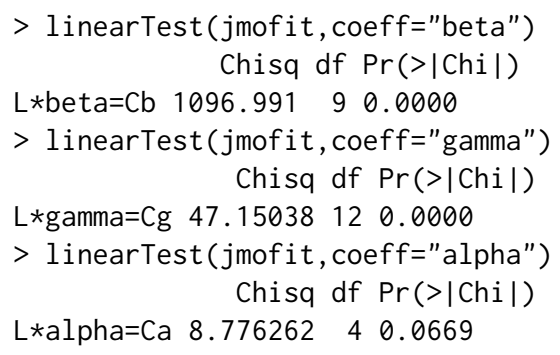

According to the $p$-values, the hypothesis $\beta_{1}=\beta_{2}=\cdots=\beta_{9}=0$ is rejected, $\gamma_{11}=\gamma_{12}=\cdots=$ $\gamma_{16}=\gamma_{21}=\gamma_{22}=\cdots=\gamma_{26}=0$ is rejected, but $\alpha_{11}=\alpha_{12}=\alpha_{21}=\alpha_{22}=0$ is not rejected at the significance level of 0.05 .

Similarly, linearTest () can be used to test a linear hypothesis for non-proportional odds fixed effects in the longitudinal submodel. For example, if we want to test $H_{0}: \alpha_{11}=\alpha_{21}$, then we can simply type:

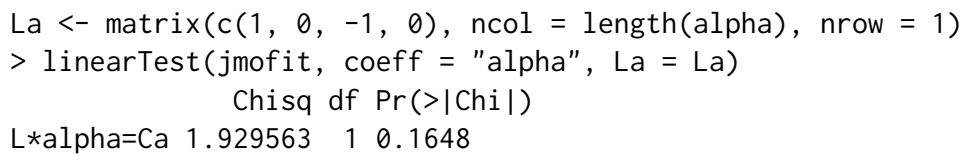

It is seen that the hypothesis $\alpha_{11}=\alpha_{21}$ is not rejected at level 0.05 .

Likewise, jmo() function allows for categorical variables. Moreover, categorical variables are allowed for setting up non-proportional odds covariates. As an illustration, here we consider the "sex" and "race" variables and use them as two of the non-proportional odds covariates. Below is another example:

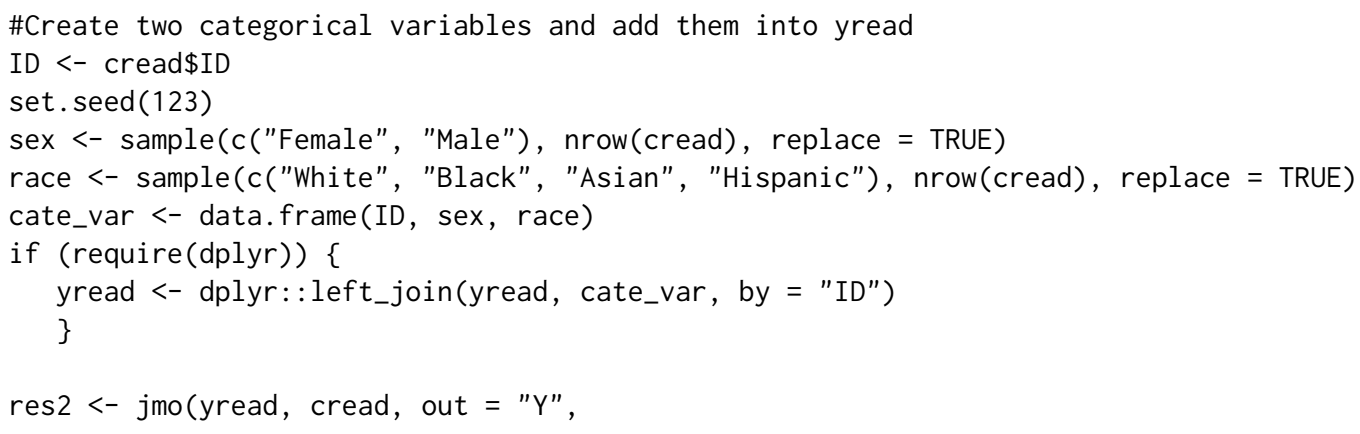




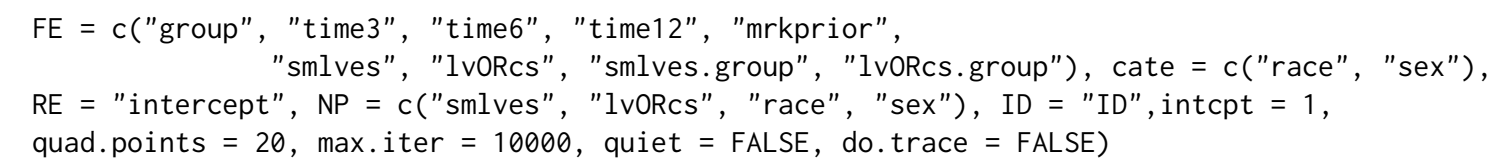

res2

Call:

jmo(long_data $=$ yread, surv_data $=$ cread, out $=$ " ${ }^{\prime}$,

FE = c("group", "time3", "time6", "time12", "mrkprior", "smlves", "lvORcs", "smlves.group", "lvORcs.group"),

RE = "intercept", NP = c("smlves", "lvORcs", "race", "sex"), ID = "ID", cate = c("race", "sex"),

intcpt $=1$, quad. points $=20$, max.iter $=10000$, quiet $=$ FALSE, do.trace $=$ FALSE)

Data Summary:

Number of observations: 1906

Number of groups: 587

Proportion of competing risks:

Risk $1: 32.88 \%$

Risk $2: 4.26 \%$

Numerical intergration:

Method: Standard Guass-Hermite quadrature

Number of quadrature points: 20

Model Type: joint modeling of longitudinal ordinal and competing risks data

Model summary:

Longitudinal process: partial proportional odds model

Event process: cause-specific Cox proportional hazard model with unspecified baseline hazard

Loglikelihood: $\quad-2271.831$

Longitudinal sub-model proportional odds: $Y \sim$ group + time $3+$ time $6+$ time12 + mrkprior + smlves + lvORcs + smlves.group + lvORcs.group + Black + Hispanic + White + Male

Longitudinal sub-model non-proportional odds: smlves_NP + lvORcs_NP + Black_NP + Hispanic_NP + White_NP + Male_NP

\begin{tabular}{lllll}
\multicolumn{1}{c}{ Longitudinal: } & Estimate & Std. Error & $95 \%$ CI & $\operatorname{Pr}(>|\mathrm{Z}|)$ \\
$\begin{array}{l}\text { Fixed effects: } \\
\text { proportional }\end{array}$ & & & & \\
group & 1.1430 & 0.1989 & $(0.7532,1.5328)$ & 0.0000 \\
time3 & 2.4607 & 0.1963 & $(2.0758,2.8455)$ & 0.0000 \\
time6 & 2.6310 & 0.1986 & $(2.2416,3.0203)$ & 0.0000 \\
time12 & 2.8717 & 0.2111 & $(2.4579,3.2854)$ & 0.0000 \\
mrkprior & -2.3329 & 0.1855 & $(-2.6965,-1.9693)$ & 0.0000 \\
smlves & 3.9941 & 0.4413 & $(3.1292,4.8589)$ & 0.0000 \\
lvORcs & -0.9469 & 0.3219 & $(-1.5778,-0.3160)$ & 0.0033 \\
smlves.group & -4.3940 & 0.7560 & $(-5.8758,-2.9123)$ & 0.0000 \\
lvORcs.group & -3.6954 & 0.4768 & $(-4.6299,-2.7608)$ & 0.0000 \\
Black & 0.8235 & 0.3162 & $(0.2038,1.4433)$ & 0.0092 \\
Hispanic & -0.0218 & 0.3289 & $(-0.6665,0.6229)$ & 0.9471 \\
White & 0.0523 & 0.3457 & $(-0.6253,0.7299)$ & 0.8797 \\
Male & -0.3528 & 0.2323 & $(-0.8080,0.1025)$ & 0.1288 \\
Non-proportional & odds: & & & \\
smlves_NP_2 & 0.3314 & 0.4310 & $(-0.5133,1.1761)$ & 0.4419 \\
lvORcs_NP_2 & -0.3148 & 0.2696 & $(-0.8432,0.2136)$ & 0.2429 \\
Black_NP_2 & 0.3781 & 0.2936 & $(-0.1973,0.9535)$ & 0.1978 \\
Hispanic_NP_2 & -0.0303 & 0.3176 & $(-0.6528,0.5923)$ & 0.9241 \\
White_NP_2 & -0.3802 & 0.3034 & $(-0.9748,0.2144)$ & 0.2102 \\
Male_NP_2 & 0.0531 & 0.2221 & $(-0.3822,0.4884)$ & 0.8110 \\
smlves_NP_3 & 2.2743 & 1.0748 & $(0.1677,4.3809)$ & 0.0343 \\
lvORcs_NP_3 & 0.0033 & 0.4632 & $(-0.9045,0.9111)$ & 0.9943 \\
Black_NP_3 & -0.2274 & 0.5419 & $(-1.2896,0.8349)$ & 0.6748 \\
Hispanic_NP_3 & -0.5070 & 0.5087 & $(-1.5040,0.4901)$ & 0.3190 \\
& & & &
\end{tabular}




$\begin{array}{lllll}\text { White_NP_3 } & 0.4205 & 0.5722 & (-0.7010,1.5420) & 0.4624 \\ \text { Male_NP_3 } & -0.8489 & 0.3911 & (-1.6155,-0.0824) & 0.0300 \\ \text { Logit-specific } & \text { intercepts: } & & & \\ \text { theta1 } & -6.0565 & 0.2868 & (-6.6186,-5.4945) & 0.0000 \\ \text { theta2 } & -4.0881 & 0.2379 & (-4.5545,-3.6217) & 0.0000 \\ \text { theta3 } & 4.1340 & 0.3437 & (3.4602,4.8077) & 0.0000\end{array}$

Survival sub-model fixed effects: Surv(surv, comprisk) group + mrkprior + smlves + lvORcs + smlves.group + lvORcs.group

\begin{tabular}{|c|c|c|c|c|c|}
\hline \multirow{2}{*}{ Survival: } & \multirow{2}{*}{ Estimate } & \multirow{2}{*}{ Std. Error } & \multirow{2}{*}{$95 \%$ CI } & \multicolumn{2}{|l|}{$\operatorname{Pr}(>|Z|)$} \\
\hline & & & & & \\
\hline \multicolumn{6}{|l|}{ Fixed effects: } \\
\hline mrkprior_1 & 0.6404 & 0.1549 & $(0.3367,0.9440)$ & 0.0000 & \\
\hline smlves_1 & -1.8107 & 0.8252 & $(-3.4280,-0.1934)$ & 0.0282 & \\
\hline lvORcs_1 & 0.4894 & 0.2450 & $(0.0092,0.9696)$ & 0.0458 & \\
\hline smlves.group_1 & 1.2608 & 1.6390 & $(-1.9517,4.4733)$ & 0.4417 & \\
\hline lvORcs.group_1 & 1.4503 & 0.4901 & $(0.4898,2.4108)$ & 0.0031 & \\
\hline group_2 & 0.2073 & 0.4831 & $(-0.7396,1.1542)$ & 0.6678 & \\
\hline mrkprior_2 & 0.0617 & 0.4343 & $(-0.7896,0.9129)$ & 0.8871 & \\
\hline smlves_2 & 0.7871 & 0.6026 & $(-0.3940,1.9683)$ & 0.1915 & \\
\hline lvORcs_2 & -0.3266 & 0.5085 & $(-1.3233,0.6701)$ & 0.5207 & \\
\hline smlves.group_2 & -0.0374 & 1.1600 & $(-2.3110,2.2362)$ & 0.9743 & \\
\hline lvORcs.group_2 & 0.0952 & 1.0591 & $(-1.9807,2.1711)$ & 0.9284 & \\
\hline \multicolumn{6}{|c|}{ Association prameter: } \\
\hline v2 & 0.0036 & 0.1577 & $(-0.3056,0.3128)$ & 0.9818 & \\
\hline \multicolumn{6}{|l|}{ Random effects: } \\
\hline sigma_b11 & 49.0241 & 5.0606 & $(39.1053,58.9430)$ & 0.0000 & \\
\hline sigma_u & 6.3475 & 1.5884 & $(3.2343,9.4607)$ & 0.0001 & \\
\hline \multicolumn{6}{|l|}{ Covariance: } \\
\hline sigma_b1u & -17.6331 & 0.7415 & $(-19.0864,-16.1797)$ & 0.0000 & \\
\hline \multicolumn{6}{|c|}{$\operatorname{coef}($ res 2, coeff $=$ "beta") } \\
\hline group & time3 & time6 & mrkprior & smlves & lvORcs \\
\hline $1.14302264 \quad 2$ & .46065107 & 2.63095850 & -2.33288371 & 3.99407491 & -0.94689649 \\
\hline smlves.group lvOF & Rcs.group & Black & Hispanic & Male & \\
\hline$-4.39403193-3$ & .69535020 & 0.82353645 & -0.02181286 & -0.35276916 & \\
\hline
\end{tabular}

\section{Older versions of jmc() and jmo()}

In the previous versions of JMcmprsk, both the previous jmc() and jmo() functions require the longitudinal input data "yfile" to be in a specific format regarding the order of the outcome variable and the random and fixed effects covariates. It also requires users to create an additional "mfile" for the longitudinal data. At the suggestions of the reviewers, in the most recent version, we focus and develop user-friendly versions of these functions.

However, for both package consistency and user's convenience, we still keep older versions of these functions in the package, and rename these functions to jmc_ $\theta()$ and jmo_ $\theta()$, respectively. Supporting functions of $j \mathrm{mo}()$ and $j \mathrm{mc}()$, such as coef(), summary (), linearTest (), also apply to jmc_ $\theta()$ and jmo_ $\theta()$ functions.

Here, we show the usage of $j m c_{-} \theta()$ with some simulated data and the "lung" data used in presenting jmc() functions.

If the data are provided as files, the function jmc_ $\theta()$ has the following usage:

library (JMcmprsk)

yfile=system.file("extdata", "jmcsimy.txt", package = "JMcmprsk")

cfile=system.file("extdata", "jmcsimc.txt", package = "JMcmprsk")

mfile=system.file("extdata", "jmcsimm.txt", package = "JMcmprsk")

jmc_ofit $=j m c_{-} \theta(p=4, y f i l e$, cfile, mfile, point $=2 \theta$, do.trace $=$ FALSE $)$

with $p$ the dimension of fixed effects (including the intercept) in yfile, the option point is the number of points used to approximate the integral in the E-step, default is 20 , and do. trace is used to 
control whether the program prints the iteration details. Additionally, the option type_file controls the type of data inputs.

If data frames or matrices are provided as inputs, we set the above type_file option as type_file $=$ FALSE in the jmc_ $\theta()$ function:

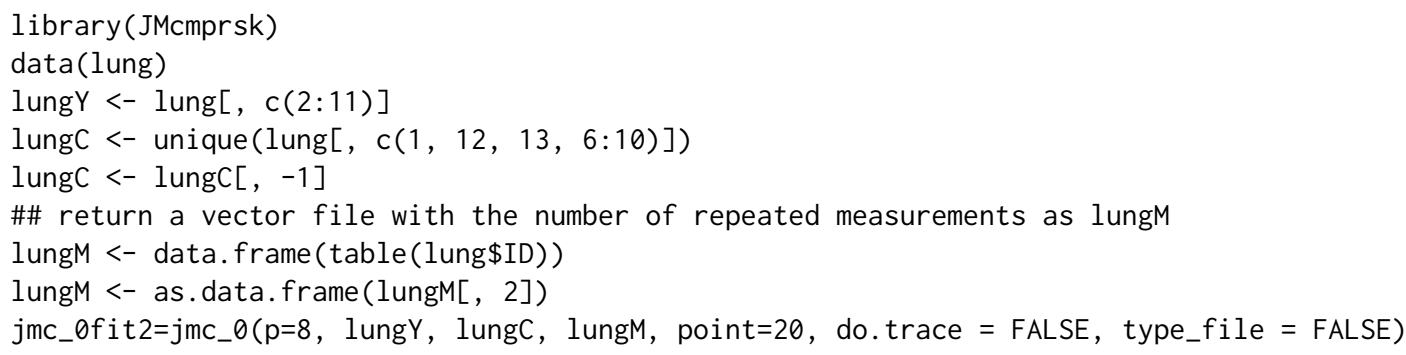

\section{Computational Complexity}

To understand the computational complexity of both jmc() and jmo() models, we carried out a variety of simulations with different sample size and different proportions of events. However, there was no clear trend observed between the proportions of events and running times. Hence, only one event distribution with different sample sizes are given here for illustration purpose. According to Figures 1 and 2, we can easily see that the run time grows much faster as sample size increases, which implies that the computational complexity does not follow a linear order. In this case, it will limit joint models to handling large and even moderate sample size data. To make the joint modeling more scalable, it is necessary to carry out a novel algorithm to reduce its computational complexity to a linear order.

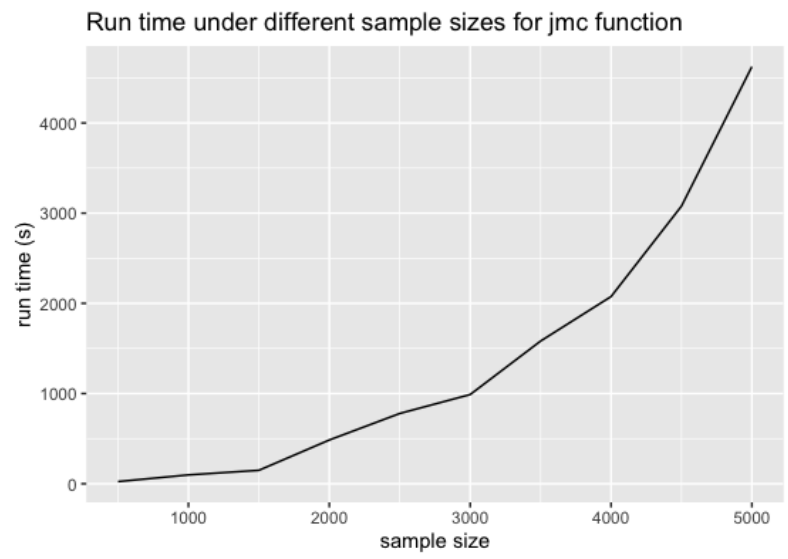

Figure 1: Run time comparison under different sample sizes for jmc() function (from 500 to 5000). Data setup: $p=4, n_{q}=6,10.4 \%$ censoring, $51.4 \%$ risk 1 , and $38.2 \%$ risk 2 . The run time under each sample size was based on one random sample.

\section{Data Simulation}

A simulation can generate datasets with exact ground truth for evaluation. Hence, the simulation of longitudinal and survival data with multiple failures associated with random effects is an important measure to assess the performance of joint modeling approaches dealing with competing risks. In JMcmprsk, simulation tools are based on the data models proposed in Elashoff et al. (2008) and Li et al. (2010), which can be used for testing joint models with continuous and ordinal longitudinal outcomes, respectively.

The main function for simulation data continuous longitudinal outcomes and survival data with multiple event outcomes is called $\operatorname{SimDataC}()$, which has the following usage:

SimDataC(k_val, p1_val, p1a_val, p2_val, g_val, truebeta, truegamma, randeffect, $y f n, c f n, m f n$ )

We briefly explain some of the important options. k_val denotes the number of subjects in study; p1_val and p1a_val denote the dimension of fixed effects and random effects in longitudinal measurements, respectively; p2_val and g_val denotes the dimension of fixed effects and number to 


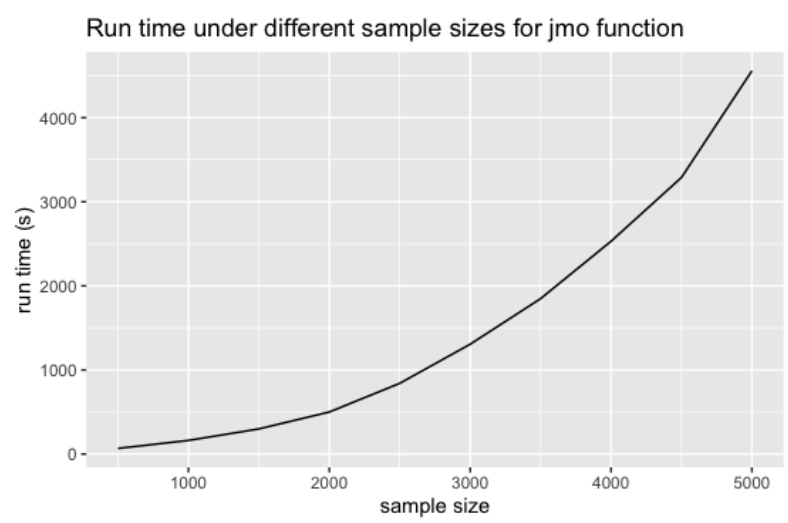

Figure 2: Run time comparison under different sample sizes for jmo() function (from 500 to 5000). Data setup: $p=4, n_{q}=10,22.4 \%$ censoring, $57.2 \%$ risk 1 , and $20.4 \%$ risk 2 . The run time under each sample size was based on one random sample.

competing risks in survival data; truebeta and truegamma represent the true values of fixe effects in the longitudinal and the survival submodels, respectively. randeffect sets the true values for random effects in longitudinal and competing risks parts, namely in the order of $\sigma, \sigma_{b}, v_{2}$, and $\sigma_{u}$.

The following example generates the datasets used in simulation study in Elashoff et al. (2008):

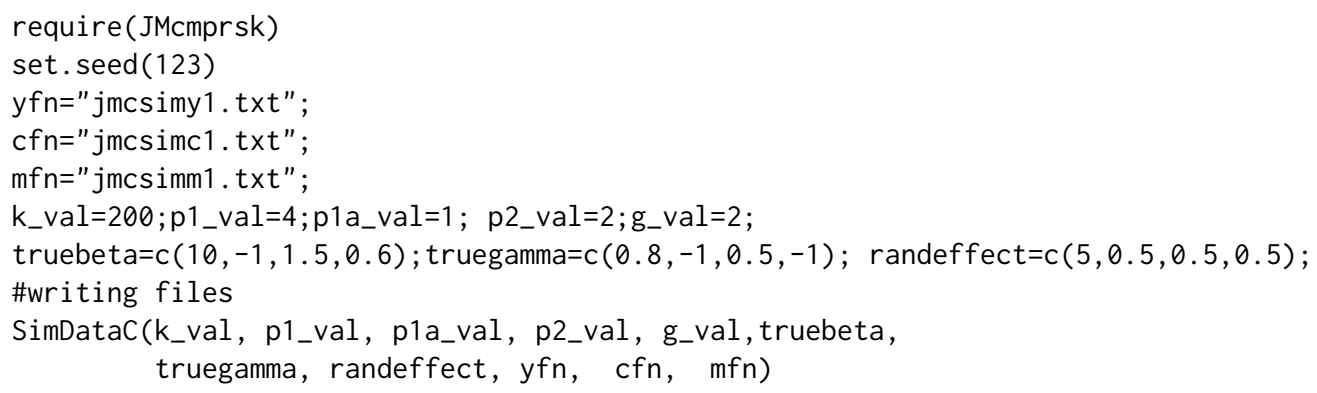

The output of function SimDataC() contains additional censoring rate information and newly generated files names for further usage.

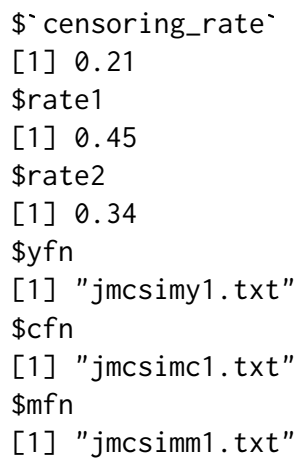

The main function for data simulation with ordinal longitudinal outcomes and survival data with multiple event outcomes is called SimDataO(), the usage of which is very similar to SimDataC():

SimData0(k_val, p1_val, p1a_val, p2_val, g_val, truebeta, truetheta, truegamma, randeffect, $y f n, c f n, m f n)$

All options have the same meanings as in $\operatorname{SimDataC}()$, while $\operatorname{SimDataO}()$ has one more option truetheta, which sets the true values of the non-proportional odds longitudinal coefficients subset.

The following example generates the datasets used in simulation study in Li et al. (2010):

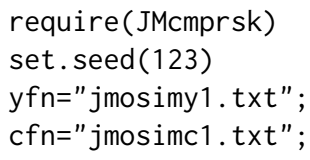




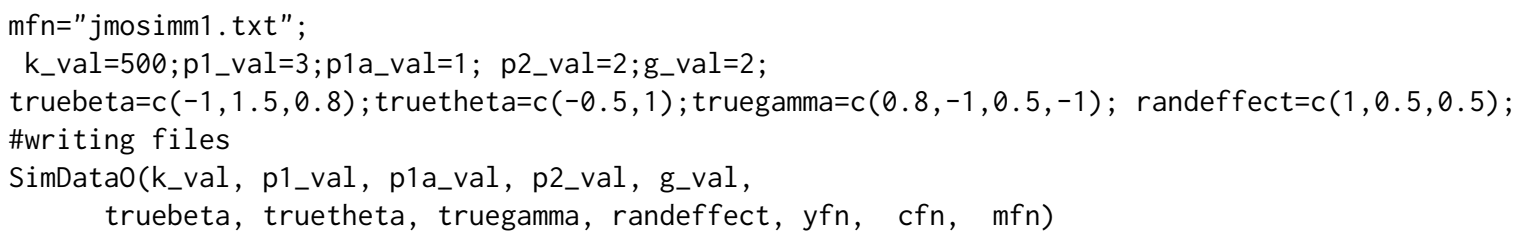

The output of the above function is

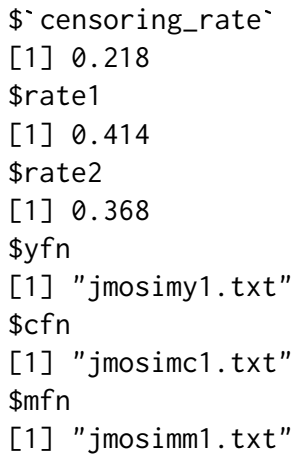

\section{Conclusions and Future Work}

In this paper, we have illustrated the capabilities of package JMcmprsk for fitting joint models of timeto-event data with competing risks for two types of longitudinal data. We also present simulation tools to generate joint model datasets under different settings. Several extensions of JMcmprsk package are planned to further expand on what is currently available. First, as the integral over the random effects becomes computationally burdensome in the case of high dimensionality, Laplace approximations or other Gauss-Hermite quadrature rules would be applied to the E-M step to speed up the computation procedure. Second, with the increasing need for predictive tools for personalized medicine, dynamic predictions for the aforementioned joint models will be added. Third, other new joint models such as joint analysis for bivariate longitudinal ordinal outcomes will be included.

\section{Acknowledgements}

We thank the reviewers for their insightful and constructive comments that led to significant improvements in our paper. The research of Hong Wang was partly supported by the National Social Science Foundation of China (17BTJ019). The research of Gang Li was partly supported by the National Institute of Health Grants P30 CA-16042, UL1TR000124-02, and P01AT003960.

\section{Bibliography}

C. Armero, C. Forné, M. Rué, A. Forte, H. Perpiñán, G. Gómez, and M. Baré. Bayesian joint ordinal and survival modeling for breast cancer risk assessment. Statistics in medicine, 35(28):5267-5282, 2016. [p53]

D. Eddelbuettel, R. François, J. Allaire, K. Ushey, Q. Kou, N. Russel, J. Chambers, and D. Bates. Rcpp: Seamless $r$ and c++ integration. Journal of Statistical Software, 40(8):1-18, 2011. [p54, 55]

R. M. Elashoff, G. Li, and N. Li. A joint model for longitudinal measurements and survival data in the presence of multiple failure types. Biometrics, 64(3):762-771, 2008. [p53, 54, 65, 66]

P. L. Ferrer. Joint modelling and prediction of several risks of cancer progression from repeated measurements of biomarkers. PhD thesis, University of Bordeaux, 2017. [p53]

M. Galassi, J. Davies, J. Theiler, B. Gough, G. Jungman, P. Alken, M. Booth, F. Rossi, and R. Ulerich. GNU scientific library. Network Theory Limited, 2002. [p54,55]

G. L. Hickey, P. Philipson, A. Jorgensen, and R. Kolamunnage-Dona. A comparison of joint models for longitudinal and competing risks data, with application to an epilepsy drug randomized controlled trial. Journal of the Royal Statistical Society: Series A (Statistics in Society), 181(4):1105-1123, 2018a. [p54] 
G. L. Hickey, P. Philipson, A. Jorgensen, and R. Kolamunnage-Dona. Joint models of longitudinal and time-to-event data with more than one event time outcome: a review. The international journal of biostatistics, 14(1), 2018b. [p53]

N. Li, R. M. Elashoff, G. Li, and J. Saver. Joint modeling of longitudinal ordinal data and competing risks survival times and analysis of the ninds rt-pa stroke trial. Statistics in medicine, 29(5):546-557, 2010. [p53, 54, 65, 66]

G. Papageorgiou, K. Mauff, A. Tomer, and D. Rizopoulos. An overview of joint modeling of timeto-event and longitudinal outcomes. Annual review of statistics and its application, 6:223-240, 2019. [p53]

C. Proust-Lima, M. Séne, J. M. Taylor, and H. Jacqmin-Gadda. Joint latent class models for longitudinal and time-to-event data: A review. Statistical methods in medical research, 23(1):74-90, 2014. [p53]

C. Proust-Lima, V. Philipps, and B. Liquet. Estimation of extended mixed models using latent classes and latent processes: The r package lcmm. Journal of Statistical Software, 78(1):1-56, 2017. [p53]

D. Rizopoulos. Joint models for longitudinal and time-to-event data: With applications in R. Chapman and Hall/CRC, 2012. [p53, 54]

N. rt PA Stroke Study. Tissue plasminogen activator for acute ischemic stroke. New England Journal of Medicine, 333(24):1581-1588, 1995. [p59]

D. P. Tashkin, R. Elashoff, P. J. Clements, J. Goldin, M. D. Roth, D. E. Furst, E. Arriola, R. Silver, C. Strange, M. Bolster, et al. Cyclophosphamide versus placebo in scleroderma lung disease. New England Journal of Medicine, 354(25):2655-2666, 2006. [p56]

A. A. Tsiatis and M. Davidian. Joint modeling of longitudinal and time-to-event data: an overview. Statistica Sinica, pages 809-834, 2004. [p53]

P. Williamson, R. Kolamunnage-Dona, P. Philipson, and A. Marson. Joint modelling of longitudinal and competing risks data. Statistics in medicine, 27(30):6426-6438, 2008. [p53, 54]

\author{
Hong Wang \\ School of Mathematics \& Statistics, Central South University \\ Changsha, Hunan Provinice, 410075 \\ China \\ wh@csu.edu.cn \\ Ning $L i$ \\ UCLA Biomathematics \\ Los Angeles, CA 90095-1772 \\ USA \\ nli@biomath. ucla.edu \\ Shanpeng $\mathrm{Li}$ \\ Department of Biostatistics,UCLA School of Public Health \\ Los Angeles, CA 90095-1772 \\ USA \\ lishanpeng0913@ucla.edu \\ Gang $L i^{*}$ \\ Department of Biostatistics,UCLA School of Public Health \\ Los Angeles, CA 90095-1772 \\ USA \\ (Corresponding Author) \\ vli@ucla.edu
}

